\title{
Depth determination of skin cancers treated with superficial brachytherapy: ultrasound vs. histopathology
}

\author{
Rosa Ballester-Sánchez, MD!, Olga Pons-Llanas, MD², Margarita Llavador-Ros, MD³, Rafael Botella-Estrada, MD, PhD', \\ Antonio Ballester-Cuñat, MD4, Alejandro Tormo-Micó, MD², Francisco Javier Celadá-Álvarez, MD², \\ Silvia Rodríguez-Villalba, MD, PhD5, Manuel Santos-Ortega, MD, PhD5, Facundo Ballester-Pallarés, MD, PhD', \\ Jose Perez-Calatayud, MD, PhD 2,5 \\ 'Dermatology Department, La Fe University and Polytechnic Hospital, Valencia, ${ }^{2}$ Radiotherapy Department, La Fe University and Polytechnic \\ Hospital, Valencia, ${ }^{3}$ Pathology Department, La Fe University and Polytechnic Hospital, Valencia, ${ }^{4}$ Radiology Department, La Fe University \\ and Polytechnic Hospital, Valencia, ${ }^{5}$ Radiotherapy Department, Benidorm Hospital, Alicante, ${ }^{6}$ Department of Atomic, Molecular and Nuclear \\ Physics, University of Valencia, Burjassot, Spain
}

\begin{abstract}
Purpose: The purpose of this study is to compare high frequency ultrasonography (HFUS) and histpathologic assessment done by punch biopsy in order to determine depth of basal cell carcinoma (BCC), in both superficial and nodular BCCs prior to brachytherapy treatment.

Material and methods: This study includes 20 patients with 10 superficial and 10 nodular BCCs. First, punch biopsy was done to confirm the diagnosis and to measure tumour depth (Breslow rate). Subsequently, HFUS was done to measure tumour depth to search for correlation of these two techniques.

Results: Neither clear tendency nor significance of the punch biopsy vs. HFUS depth determination is observed. Depth value differences with both modalities resulted patient dependent and then consequence of its uncertainty. Conceptually, HFUS should determine the macroscopic lesion (gross tumour volume - GTV), while punch biopsy is able to detect the microscopic extension (clinical target volume - CTV). Uncertainties of HFUS are difficult to address, while punch biopsy is done just on a small lesion section, not necessarily the deepest one.

Conclusions: According to the results, HFUS is less accurate at very shallow depths. Nodular cases present higher depth determination differences than superficial ones. In our clinical practice, we decided to prescribe at $3 \mathrm{~mm}$ depth
\end{abstract} when HFUS measurements give depth lesion values smaller than this value.

J Contemp Brachytherapy 2014; 6, 4: 356-361 DOI: $10.5114 / j \mathrm{cb} .2014 .47860$

Key words: basal cell carcinoma, electronic brachytherapy, punch biopsy, skin brachytherapy, skin cancer, ultrasound.

\section{Purpose}

Basal cell carcinoma (BCC) is a common skin cancer arising from the basal layer of the epidermis and its appendages. It is particularly common in Caucasian people, increases with age and is basically related to exposure to ultraviolet radiation [1]. The incidence of BCC is increasing worldwide [1]. It is a malignant locally invasive epidermal tumour with a good prognosis due to a slow growth-rate and low metastatic potential. Local invasion and tissue destruction, however, cause patient morbidity.

There are several clinical and histopathologic types of BCC. The most common types are nodular and superficial BCC, which occur for the most part on the face [1]. Less frequent types include micronodular, morpheaform, and basoesquamous cell carcinomas. The diagnosis of BCC is made clinically, aided by dermoscopy. A skin biopsy is usually also performed to provide histological confirmation. Once the diagnosis is established, appropriate treatment offers a high probability of cure. The patient does, however, have an increased risk of additional skin malignancies. The choice of appropriate therapy is dependent upon the characteristics of the lesion and patient-specific factors. Treatment modalities include electrodesiccation and curettage, cryotherapy, surgical excision including Mohs surgery, topical 5-fluouracil or imiquimod, photodynamic therapy, and radiotherapy [2-5].

Determining tumour extension, and defining accurate lateral and deep safety margins are very important aspects in the treatment approach for BCC. It is not possible to determine lesion depth based on clinical observations alone, because there might be an overestimation of the extension,
Address for correspondence: Rosa Ballester-Sánchez, MD, Dermatology Department, La Fe University and Polytechnic Hospital, Valencia, Spain, Bulevar sur s/n, 46026 Valencia, Spain, phone: +34 961244186, e-mail: roseta999@hotmail.com
Received: 15.09 .2014

Accepted: 08.11 .2014

Published: 30.12.2014 
which may lead to unnecessary tissue excision or radiation. This in turn could then result in aesthetic problems for the patient [6]. In addition, the rate of incomplete excision of BCCs has been reported to be 5-25\% [7-14]. Although surgery is the first-line treatment for nonmelanoma skin cancers, radiotherapy can be indicated in selected cases. When radiotherapy is the treatment of choice, brachytherapy (BT) may be a good option for shallow, widespread lesions, or lesions on anatomic sites (e.g., hand, full scalp) that lie immediately above structures, which are vulnerable to irradiation [15]. High-dose-rate (HDR) BT approaches offer significant advantages in this setting due to adaptability, patient protection, and variable dose fractionation schedules, and achieve excellent cure rates and cosmetic results [16]. Several innovative applicators have been introduced to the BT community, and the use of skin BT has significantly increased over the years. The Valencia applicator [17-20] (Nucletron, an Elekta company, Elekta $\mathrm{AB}$, Stockholm, Sweden) is a new superficial device that improves the dose distribution compared with that of the Leipzig applicator [21-24] (Elekta, Stockholm, Sweden and Varian Medical Systems, Palo Alto, CA, USA). Recently, electronic brachytherapy using specific applicators has also become available, as Xoft $[15,25]$ (Xoft Inc., San Jose, CA, USA) and Esteya [26] (Nucletron, an Elekta company, Elekta AB, Stockholm, Sweden).

Brachytherapy provides minimal dose delivery to surrounding healthy tissue, thus enabling good functional and cosmetic results. Brachytherapy appears to be most effective for small, primary, and/or superficial squamous cell carcinomas and basal cell carcinomas, where it is associated with excellent cosmetic results. The primary benefit of BT compared to external beam radiation therapy is the ability of BT to deliver radiation to the target tissue, with less injury to surrounding normal-appearing skin.

Lateral and deep tumour delimitations are the main challenges when treating basal cell carcinomas with BT. Lateral delimitation may be aided by dermoscopy [27], and deep demarcation can be estimated by biopsy and/ or imaging techniques. In superficial BT, the dose is prescribed to the deepest point of the target, which results in a higher dose between the source and this prescription point $[20,28]$. A punch biopsy provides confirmation of the tumour's histopathology as well as determining its depth. However, it is an invasive technique, which only measures the depth in a portion of the tumour which cannot be representative. Ultrasonography, on the other hand, is a non-invasive, painless, non-ionizing, low risk, and non-expensive method, which is of academic interest in diagnosing BCC [29]. In normal skin, the dermis is markedly echogenic and sharply demarcated from hypoechogenic subcutaneous fat [30]. Basal cell carcinomas will appear more hypoechogenic than adjacent, normal dermis due to a medium change. The use of high frequency ultrasonography (HFUS) between $10 \mathrm{MHz}$ and $50 \mathrm{MHz}$ has made it possible to visualize deep layers of skin, and to define very small hypo-echoic masses. Using the refraction of ultrasonography waves at the interface between the perilesional hyper-echoic area and the hypoechoic area of the tumour itself, it is possible to precisely define the lesion [31].
High frequency ultrasonography has been shown to be potentially quite useful in BCC for both tumour measurement (for planning surgical resection) [32] and as a diagnostic technique $[33,34]$. Most of the published research in this field deals with the study of tumour size, delineation of surgical margins, and comparison of ultrasound findings with histologic results obtained following subsequent excisional biopsy of the lesion [35-39]. Concordance rates between HFUS findings and histology results for tumour size are between 73-98\% [34]. Published rates of tumour-free margins assessed by HFUS are as high as $95 \%$ [40], but this has never been studied prior to radiotherapy treatment.

The purpose of this study is to compare both HFUS and punch biopsy methods in determining the depth of basal cell carcinomas prior to brachytherapy. We also present the strategy adopted at our department as a result of this present study.

\section{Material and methods}

\section{Patients}

This study included 10 men and 10 women, all of them Caucasian, with 10 superficial and 10 nodular BCCs. All tumours were primary, maximum $20 \mathrm{~mm}$ in diameter and were located in a regular or flat area that was not adjacent to or over a burn, scar or inflammatory process. Only clinically apparent nodular and superficial BCCs were included in this study. Exclusion criteria included other BCC varieties, recurrent BCCs, and BCCs that were in locations difficult to image or treat with isotope or electronic brachytherapy applicators. All lesions were studied by histopathology and HFUS to determine tumour depth (Breslow thickness) prior to BT treatment. The mean time between the two techniques was 53 days (range 30-92 days). In the first instance, a punch biopsy was taken. This technique allowed us to confirm the diagnosis of BCC and to measure the microscopic depth of the tumour that represents the clinical target volume (CTV) depth. Subsequently, HFUS imaging was done to measure the macroscopic depth of the tumour, which represents the gross tumour volume (GTV) depth. In every case, HFUS was done at least one month after the biopsy in order to avoid peritumoral inflammation due to the biopsy scar.

To investigate whether HFUS is sufficient to determine a correct prescription depth dose, the correlation between both techniques was studied. This study was conducted under Helsinki II ethical principles after approval by the Medical Ethics Committee at our hospital.

\section{Punch biopsy technique. Histopathology}

A $3 \mathrm{~mm}$ diameter punch biopsy, including the whole dermis, was performed in all lesions. The deepest site estimated clinically was the site chosen for the biopsy; this is the usual method practised by dermatologists. The more nodular part usually corresponds to the deepest part of the tumour. An intralesional injection of mepivacaine was administered prior to the biopsy and a silk suture was used to close the wound. Histopathologic 


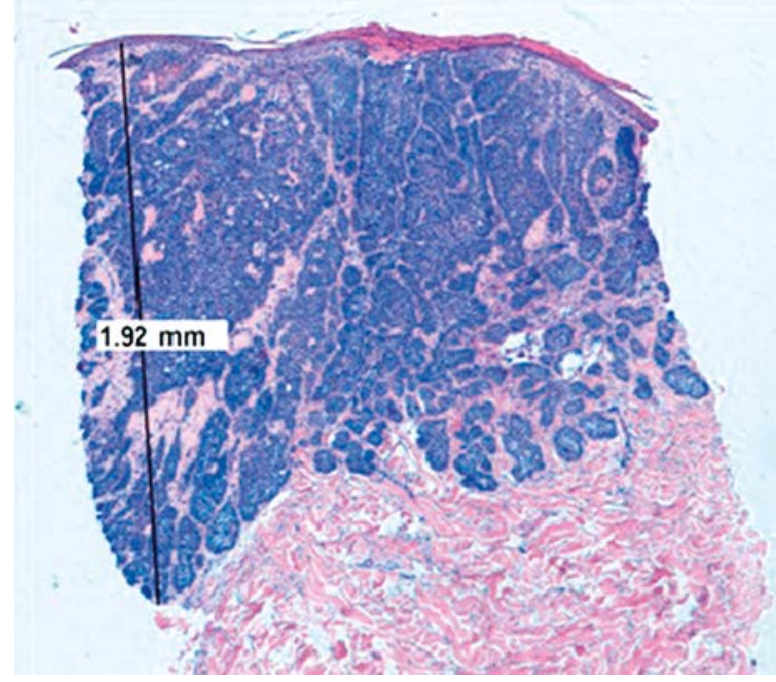

Fig. 1. Example of depth histopathologic assessment (Breslow rate) using a Leica DMD108 digital microimaging network

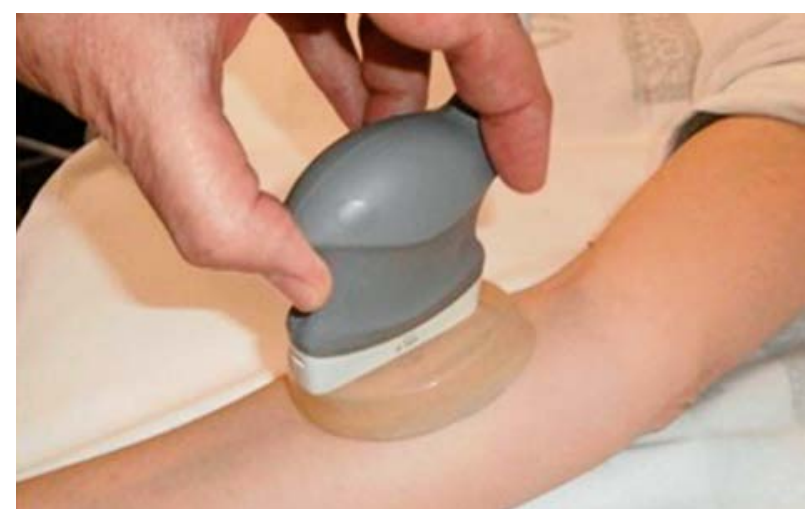

Fig. 2. Illustration of the probe plus gel pad use during the acquisition

assessment of depth was done with the Leica DMD108 digital microimaging network (Leica Microsystems SLU, Barcelona, Spain). Tumour thickness was measured from the granular layer to the deepest portion of the tumour, as shown in Figure 1.
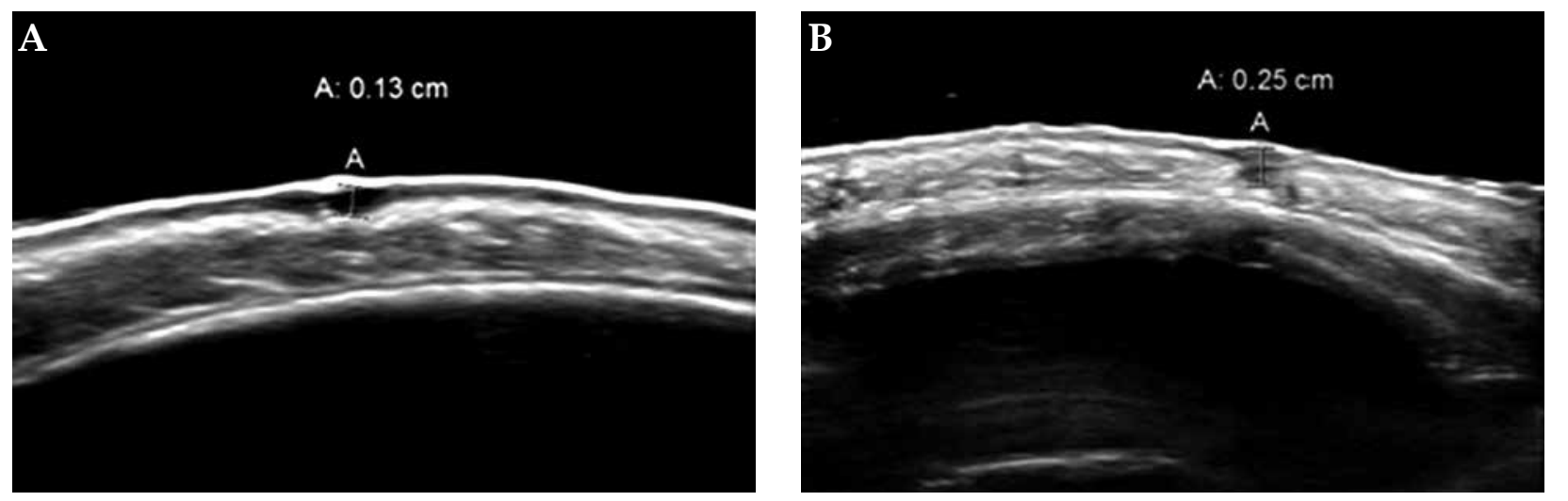

Fig. 3. High frequency ultrasonography examples of depth measurement. A) Superficial basal cell carcinoma. B) Nodular basal cell carcinoma

\section{High frequency ultrasound imaging}

After the biopsy, a radiologist who was an expert in skin lesions, estimated the depth of the lesions. All BCCs were scanned in vivo using a high resolution B-scan with an $18 \mathrm{MHz}$ hand-held transducer (Siemens Acuson S2000, Munich, Germany). A $2 \mathrm{~cm} \times 9 \mathrm{~cm}$ gel pad (Aquaflex, Pallejà, Barcelona, Spain) was applied over the skin to enhance the air-skin interface (Fig. 2). High frequencies have better resolution, but lower frequencies are often used in hospital, and it has been reported in the literature that there is a good correlation between ultrasonic and histologic measurements (with complete lesion excision), even with probes emitting frequencies of $15 \mathrm{MHz}$ or lower [34]. In each lesion, the depth (from the epidermal surface to the deepest hypo-echoic point of the tumour) was measured. Because the epidermis thickness is approximately $0.1 \mathrm{~mm}$, when HFUS did not show any value, $0.1 \mathrm{~mm}$ was assigned. Examples are given in Figure 3 for both superficial and nodular lesions.

\section{Results}

The clinical and histological characteristics of the lesions of the 20 patients studied are presented in Table 1. There were 10 men and 10 women, with 10 superficial and 10 nodular BCCs. The mean age of the patients was 67 years (range 51-89 years). Fifteen lesions were located on the face and 5 on the trunk. Resulting lesion depths with HFUS and Breslow are presented in Figure 4 for both superficial and nodular lesions, respectively. In the superficial lesions, the Breslow rate was similar or higher than HFUS in most cases $(8 / 10)$. In the nodular lesions, however, there was no clear trend. The largest difference between the two techniques was $2.7 \mathrm{~mm}$.

Statistical analyses were performed calculating covariance and correlation matrices for the HFUS and Breslow depths. A value of $p=0.05007>0.05$ was found. So, although it could be considered that some correlation exists for the scatter plot in Figure 4, it does not show any clear dependence between both variables. We also found no correlation when the different types of BCC were analysed separately. 
Table 1. Clinical and histological characteristics

\begin{tabular}{|c|c|c|c|c|c|c|}
\hline Tumour & Sex & Age (years) & Histological subtype & Location & HFUS (mm) & Breslow $(\mathrm{mm})$ \\
\hline 1 & Male & 65 & Superficial & Preauricular & 0.1 & 1.0 \\
\hline 2 & Female & 75 & Nodular & Retroauricular & 1.5 & 1.1 \\
\hline 3 & Male & 89 & Nodular & Forehead & 1.3 & 1.4 \\
\hline 4 & Male & 88 & Nodular & Preauricular & 0.1 & 2.8 \\
\hline 5 & Male & 63 & Superficial & Trunk & 0.1 & 0.5 \\
\hline 6 & Female & 51 & Nodular & Glabellar & 2.7 & 2.3 \\
\hline 7 & Male & 70 & Nodular & Cheek & 3.7 & 2.6 \\
\hline 8 & Female & 80 & Nodular & Nose & 1.3 & 1.8 \\
\hline 9 & Male & 70 & Superficial & Trunk & 0.1 & 0.3 \\
\hline 10 & Female & 59 & Superficial & Trunk & 0.1 & 0.3 \\
\hline 11 & Male & 56 & Superficial & Trunk & 0.1 & 0.3 \\
\hline 12 & Male & 67 & Superficial & Cheek & 0.1 & 0.8 \\
\hline 13 & Female & 57 & Nodular & Forehead & 2.0 & 3.1 \\
\hline 14 & Male & 67 & Nodular & Forehead & 1.6 & 1.4 \\
\hline 15 & Female & 56 & Nodular & Forehead & 1.6 & 3.2 \\
\hline 16 & Female & 66 & Superficial & Trunk & 0.1 & 1.9 \\
\hline 17 & Female & 57 & Superficial & Cheek & 0.1 & 0.5 \\
\hline 18 & Male & 84 & Nodular & Forehead & 1.3 & 1.2 \\
\hline 19 & Female & 73 & Superficial & Forehead & 1.1 & 1.3 \\
\hline 20 & Female & 81 & Superficial & Forehead & 1.3 & 0.8 \\
\hline
\end{tabular}

HFUS - high frequency ultrasonography
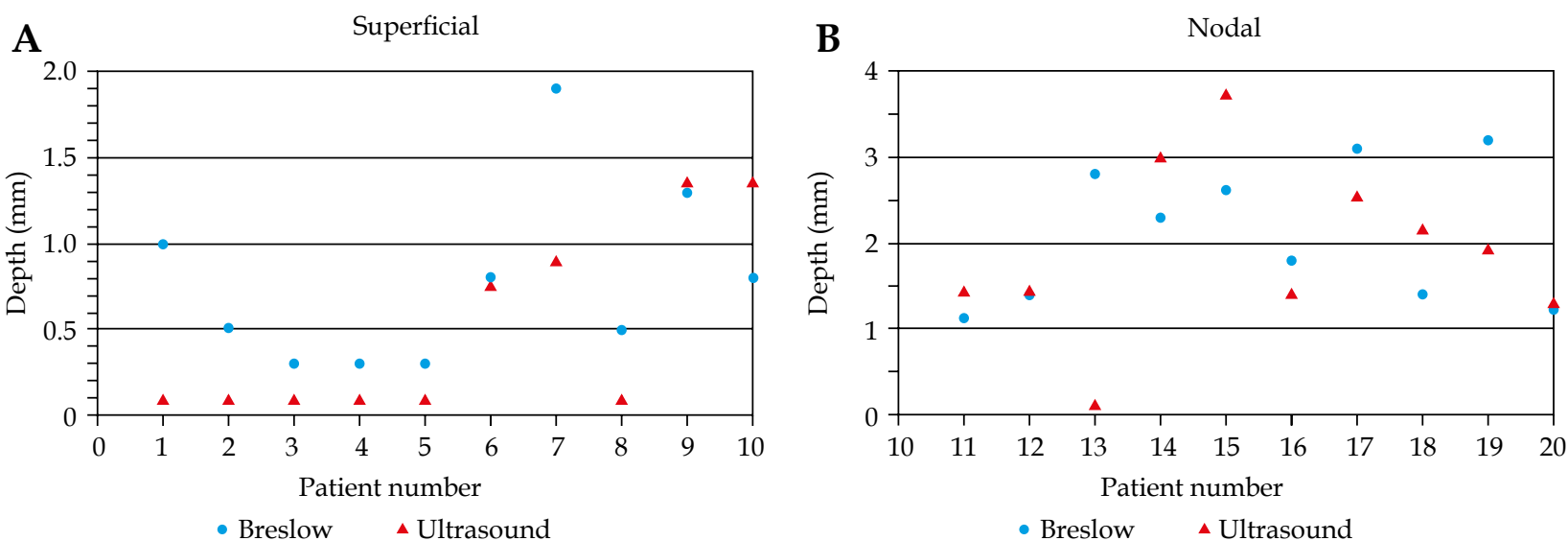

Fig. 4. A) Histopathology (Breslow rate) vs. high frequency ultrasonography (HFUS) depth determination for the 10 patients evaluated with superficial basal cell carcinoma (BCC). B) The same but with nodular BCC

\section{Discussion}

Conceptually, HFUS should determine the GTV while histopathology is able to detect the CTV. Uncertainty of HFUS depth measurements are difficult to address. Histopathologic measurements are done just on a small lesion section, which is not necessarily the deepest one. There are some limitations in the use of HFUS in BCC. The large ultrasound probe makes access difficult in certain tumour locations, although brachytherapy is also not typically used in these locations anyway. Assessment with HFUS can also be difficult in the vicinity of scars. Small tumour aggregates are not detected by HFUS and it is also not possible to differentiate between the tumour and adjacent in- 
flammation. Furthermore, HFUS is an operator-dependent technique. On the other hand, HFUS has an important advantage over punch-biopsy as it allows a three-dimensional analysis of the tumour, whereas clinical measurements only permit a two-dimensional view [40].

In this study, all US acquisitions were done by the same radiologist. In order to explore the intraobserver variability, the images were reviewed by the radiologist 3 times with a sufficient time interval in between. The resulting differences were negligible. According to the results, HFUS was less accurate at very shallow depths. The nodular cases presented with larger depth differences than the superficial ones. In most cases of superficial BCCs, the HFUS depth measurement was lower than the histopathologic one, which was in contrast to the nodular cases. Neither clear tendency nor significance was observed from this depth comparison after applying standard statistical tests to search for depth measurement correlations between the two techniques.

\section{Limitations in our study}

We used an $18 \mathrm{MHz}$ ultrasound probe. High frequencies have better resolution, but lower frequencies are usually used in hospital. It has been reported in the literature that there is a good correlations between ultrasonic and histologic measurements, even with probes emitting frequencies of $15 \mathrm{MHz}$ or lower [34]. In our study, punch biopsy was done prior to HFUS to confirm the diagnosis before measuring the tumour depth. This can lead to two problems: the biopsy could potentially remove the deepest part of the tumour, and both the scar and the inflammation after biopsy could distort/change the echographic image. Trying to avoid the latter problem, ultrasound was performed at least one month after the biopsy. There were a limited number of patients (20) included in this study. However, the number is sufficient to demonstrate that there is no clear correlation between these two methods.

\section{Conclusions}

High frequency ultrasonography vs. histopathologic depth determination have been compared for 10 superficial and 10 nodular basal cell carcinomas. Neither a clear trend nor a significant difference in histopathology compared to HFUS depth determination was observed. As a result of: 1) the comparison results of the present study, 2) the depth dose gradient, 3) the maximum skin dose using radionuclide applicators or electronic BT, and 4) the cosmesis experienced in clinical practice, we have decided in our protocol to prescribe to $3 \mathrm{~mm}$ depth when HFUS measurements give lesion depths smaller than this threshold depth.

\section{Acknowledgments}

This study was supported within a collaborative project with Elekta Brachytherapy (Elekta Company, Veenendaal, The Netherlands). This study was also partially supported by Generalitat Valenciana (Project PROMETEOII/ 2013/010) and by Spanish Government under Project No. FIS2013-42156.

\section{Disclosure}

Authors report no conflict of interest.

\section{References}

1. Lear W, Dahlke E, Murray CA. Basal cell carcinoma: review of epidemiology, pathogenesis, and associated risk factors. J Cutan Med Surg 2007; 11: 19-30.

2. Ormerod A, Rajpara S, Craig F. Basal cell carcinoma. Clin Evid 2010; 6: 1719.

3. Fimhaber JM. Diagnosis and treatment of basal cell and squamous cell carcinoma. Am Fam Physician 2012; 86: 161-168.

4. Roozeboom MH, Arits AH, Nelemans PJ et al. Overall treatment success after treatment of primary superficial basal cell carcinoma: a systematic review and meta-analysis of randomized and nonrandomized trials. Br J Dermatol 2012; 167: 733-756.

5. Bahner JD, Bordeaux JS. Non-melanoma skin cancers: photodynamic therapy, cryotherapy, 5-fluorouracil, imiquimod, diclofenac, or what? Facts and controversies. Clin Dermatol 2013; 31: 792-798.

6. Bobadilla F, Wortsman X, Munoz C et al. Pre-surgical high resolution ultrasound of facial basal cell carcinoma: correlation with histology. Cancer Imaging 2008; 22: 163-172.

7. Griffiths RW. Audit of histologically incompletely excised basal cell carcinomas: recommendations for management by re-excision. Br J Plast Surg 1999; 52: 24-28.

8. Kumar P, Orton CI, McWilliam LJ et al. Incidence of incomplete excision in surgically treated basal cell carcinoma: a retrospective clinical audit. Br J Plast Surg 2000; 53: 563-566.

9. Nagore E, Grau C, Molinero J et al. Positive margins in basal cell carcinoma: relationship to clinical features and recurrence risk. A retrospective study of 248 patients. J Eur Acad Dermatol Venereol 2003; 17: 167-170.

10. Bogdanov-Berezovsky A, Cohen AD, Glesinger R et al. Risk factors for incomplete excision of basal cell carcinomas. Acta Derm Venereol 2004; 84: 44-47.

11. Bogelund FS, Philipsen PA, Gniadecki R. Factors affecting the recurrence rate of basal cell carcinoma. Acta Dermatol Venereol 2007; 87: 330-334.

12. Farhi D, Dupin N, Palangie A et al. Incomplete excision of basal cell carcinoma: rate and associated factors among 362 consecutive cases. Dermatol Surg 2007; 33: 1207-1214.

13. Su SY, Giorlando F, Ek EW et al. Incomplete excision of basal cell carcinoma: a prospective trial. Plast Reconstr Surg 2007; 120: $1240-1248$.

14. Fargnoli MC, Kostaki D, Piccioni A et al. Dermoscopy in the diagnosis and management of non-melanoma skin cancers. Eur J Dermatol 2012; 22: 456-463.

15. Alam M, Nanda S, Mittal B et al. The use of brachytherapy in the treatment of nonmelanoma skin cancer: a review. J Am Acad Dermatol 2011; 65: 377-388.

16. National Comprehensive Cancer Network guidelines 2013. http://www.nccn.org/professionals/physician_gls/f_guidelines.asp

17. Granero D, Perez-Calatayud J, Jimeno J et al. Design and evaluation of a HDR skin applicator with flattening filter. Med Phys 2008; 35: 495-503.

18. Lliso F, Granero D, Perez-Calatayud J et al. Dosimetric evaluation of internal shielding in a high dose rate skin applicator. J Contemp Brachytherapy 2011; 3: 32-35.

19. Granero D, Perez-Calatayud J, Ballester F et al. Radiation leakage study for the Valencia applicators. Physica Medica 2013; 29: 60-64.

20. Tormo A, Celada F, Rodriguez S et al. Non-melanoma skin cancer treated with HDR Valencia applicator: Clinical outcomes. J Contemp Brachytherapy 2014; 6: 167-172. 
21. Evans MDC, Yassa M, Podgorsak EB et al. Surface applicators for high dose rate brachytherapy in aids-related Kaposi sarcoma. In J Radiat Oncol Biol Phys 1997; 39: 769-774.

22. Hwang IM, Lin SY, Lin LC et al. Alternative effective modality of Leipzig applicator with an electron beam for the treatment of superficial malignancies. Nuc Inst Meth A 2003; 508: 460-466.

23. Niu H, His WC, Chu JCH et al. Dosimetric characteristics of the Leipzig surface applicators used in the high dose rate brachy radiotherapy. Med Phys 2004; 31: 3372-3377.

24. Perez-Calatayud J, Granero D, Ballester F et al. A dosimetric study of the Leipzig applicators. Int J Rad Oncol Biol Phys 2005; 62: 579-584.

25. Rong Y, Welsh JS. Surface applicator calibration and commissioning of an electronic brachytherapy system for nonmelanoma skin cancer and treatment. Med Phys 2010; 37: 5509-5517.

26. Garcia-Martinez T, Chan J-P, Perez-Calatayud J et al. Dosimetric characteristics of a new unit for electronic skin brachytherapy. J Contemp Brachytherapy 2014; 6: 1-9.

27. Ballester R, Pons O, Pérez J, Botella R. Dermoscopy margin delineation in radiotherapy planning for superficial and nodular basal cell carcinoma. Br J Dermatol 2014 [In press].

28. Berridge JK, Morgan DA. A comparison of late cosmetic results following two different radiotherapy techniques for treating basal cell carcinoma. Clin Oncol (R Coll Radiol) 1997; 9: 400-402.

29. Telfer NR, Colver GB, Morton CA. British Association of Dermatologists. Guidelines for the management of basal cell carcinoma. Br J Dermatol 2008; 159: 35-48.

30. Hedrick WR, Hykes DL, Starchman DE. Ultrasound physics and instrumentation. $4^{\text {th }}$ ed. Elsevier Mosby-Year Book, St. Louis 2005.

31. Schmid-Wendtner MH, Dill-Muller D. Ultrasound technology in dermatology. Semin Cutan Med Surg 2008; 27: 44-51.

32. Caresana G, Giardini R. Dermoscopy-guided surgery in basal cell carcinoma. J Eur Acad Dermatol Venereol 2010; 24: 1395 1399.

33. Wortsman X, Jemec GBE. High resolution ultrasound applications in dermatology. Rev Chilena Dermatol 2006; 22: 37-45.

34. Hernández C, del Boz J, de Troya M. Can high-frequency skin ultrasoud be used for the diagnosis and management of basal cell carcinoma? Actas Dermosifiliogr 2014; 105: 107-111.

35. Gupta AK, Turnbull DH, Foster FS et al. High frequency 40 $\mathrm{MHz}$ ultrasound: a possible noninvasive method for the assessment of the boundary of basal cell carcinomas. Dermatol Surg 1996; 22: 131-136.

36. Lassau N, Spatz A, Avril MF et al. Value of high-frequency US for preoperative assessment of skin tumors. Radiographics 1997; 17: 1559-1565.

37. Jovanovic DL, Katic V, Jovanovic B. Value of preoperative determination of skin tumors thickness with $20-\mathrm{MHz}$ ultrasound. Arch Dermatol 2005; 141: 269-270.

38. Jambusaria-Pahlajani A, Schmults CD, Miller CJ et al. Test characteristics of high-resolution ultra-sound in the preoperative assessment of margins of basal cell and squamous cell carcinoma in patients undergoing Mohs micrographic surgery. Dermatol Surg 2009; 35: 9-15.

39. Nassiri-Kashani M, Sadr B, Fanian F et al. Pre-operative assessment of basal cell carcinoma dimensions using high frequency ultra-sound and its correlation with histopathology. Skin Res Technol 2013; 19: 132-138.

40. Desai T, Desai A, Horowitz D et al. High-frequency ultrasound in the evaluation of superficial and nodular basal cell carcinomas. Dermatol Surg 2007; 33: 1220-1227. 